\title{
Comparative study between ischemic compression and dry needling in myofascial pain syndrome: possibilities in health
}

\author{
Vanessa Renata Molinero de Paula', Gustavo Melo de Paula', Jadson Justi², Cláudia F. Soares', \\ Hugo Machado Sanchez ${ }^{3}$
}

\begin{abstract}
Introduction: Myofascial Pain Syndrome (MPS) is a neuromuscular regional dysfunction characterized by the presence of sensitive regions in strained muscle bands (trigger points), able to cause local and referred pain, functional losses and change the quality of life (QOL) of affected people. It can be triggered by musculoskeletal injuries, postural imbalances, endocrinal factors and psych emotional disorders. The diagnostic is clinical, the intervention is multidisciplinary and the treatment may involve the use of drugs and several invasive and non-invasive techniques. Objective: Compare the effects of ischemic compression associated to physiotherapy and dry needling associated to pain control and the QOL of people with MPS. Methods: 40 subjects took part of the study. They were of both genders, aged from 20 to 60 years old, with MPS, divided into 2 groups with 20 participants each. The first group receives ischemic compression and physiotherapy and the second, dry needling and physiotherapy. The statistical analysis involves proportions for categorical variables, significance and standard deviation for continuous variables. In order to evaluate the change in the pain and QOL perception of subjects, the Student's $t$-test $(p<0.05)$. Results: The best results were verified in the dry needling and physiotherapy group. Conclusion: Both ischemic compression and physiotherapy as dry needling and physiotherapy can promote pain relief and better QOL in subjects suffering from MPS.
\end{abstract}

Keywords: Health; Pain; Therapeutic Treatment; Quality of Life; Physiotherapy.

\section{INTRODUCTION}

Myofascial Pain Syndrome (MPS) is currently defined as a regional neuromuscular dysfunction characterized by the presence of sensitive regions in contracted / strained muscle bands (nodules), also called trigger points (TP), capable of causing local and referred pain, with specific pathways, impairment of joint movement, decreased muscular strength, loss of performance at work, sports and daily life activities, with impairment of quality of life $(\mathrm{QOL})^{(1)}$. In addition to local or referred pain, MPS may also be associated with autonomic muscle phenomena, such as: vasoconstriction, sweating and piloerection, and proprioceptive disorders such as imbalance, dizziness, tinnitus, and weight distortion of objects ${ }^{(2)}$. TP can be classified as latent or active and can be perceived through palpation of muscular nodules ${ }^{(3)}$. The latent TP does not cause pain, whereas the active TP produces symptomatic irritability in the muscle or fascia, with referred pain pattern associated with the activation of muscle nociceptors, which occurs through the intense release of endogenous neuropeptide-like substances derived from arachidonic acid and inflammatory mediators ${ }^{(4)}$. The TP develop in the myofascial, mainly at the most central point of the muscular womb, where the primary or central motor plate is located. However, secondary TP or satellites, with smaller volume (nodulation), develop in response to primary TP. These satellite points usually develop along the tension lines. External factors such as age, body morphology, posture, weight gain and congenital malformations can be decisive in the formation and activation of TP(4).

Although it is a common condition, particularly in pain centers, in orthopedics and traumatology, physiatry, rheumatology and neurology clinics, MPS is often neglected or misdiagnosed by many health professionals, being one of the most common causes of muscle pain and disability in patients presenting with this type of symptoms, being more evident in an age group between 31 and 50 years old ${ }^{(5)}$. MPS can trigger acute or chronic pain from the presence of active TP in several muscles, which are sensitized to compression and stretching, causing localized and referred pain, that is, in distant paths of $\mathrm{TP}^{(6)}$. MPS can be triggered by several factors, such as trauma, excessive use, infection, inflammation, postural changes, acute myositis, endocrine imbalances, prolonged exposure to cold, emotional stress, joint and neural dysfunctions $s^{(7)}$. It is estimated that $90.0 \%$ of people will present MPS at some stage of their life and that, currently, this condition reaches about $30.0 \%$ of the pain condition in patients attended in a general clinic and $85.0 \%$ 
of the patients admitted at the pain centers ${ }^{(8)}$. The diagnosis of MPS involves the patient's history and physical examination, ruling out the need for complementary tests ${ }^{(9)}$. There is a description of painful condition, strained muscle bands and loss of functionality or muscle performance. During palpation, there is the presence of active TP, which may be associated with edema, a sign of withdrawal due to the presence of local and referred pain (jump sign), palpable tense muscle band and local contractile response (twitch response). MPS therapeutics becomes complex as its diagnosis is often neglected and not known to many health professionals, being assertive in a few cases $^{(10)}$. MPS that is not diagnosed early and treated properly may become chronic, disabling, and constitute factor of financial loss for people affected, not to mention that this condition, isolated or associated with other lesions and or pathologies of the musculoskeletal system, has been considered one of the most common causes of work leave ${ }^{(11)}$.

The lack of identification or misdiagnosis of MPS by many health professionals, who are still unaware of this condition, culminates in the indication of inadequate therapies that do not eliminate the chronic pain characteristic of this syndrome, resulting in loss of productivity, psychoemotional disorders and increase of postural compensations ${ }^{(4)}$. Many patients with MPS end up being labeled as neurotic or having psychosomatic abnormalities, without specific diagnosis ${ }^{(12)}$. After the diagnosis of MPS has been established, several types of treatments can be used, all of them aiming at the deactivation of the active TP, restoring the normal length of the muscle, promoting muscular relaxation and adequate local vascularization, restoration of the motion range, flexibility and muscle strength ${ }^{(13)}$. In addition, patient orientation is necessary in order to avoid the precipitating and / or perpetuating factors of this condition to prevent relapses $^{(10)}$. The treatment of MPS is vast and may involve several therapies, either isolated or in combination ${ }^{(14)}$. Analgesic and anti-inflammatory drugs are important in pain control and aid in kinesiotherapy. However, its chronic use, in cases of MPS has not been shown to be effective. Low-dose tricyclic antidepressants and specific inhibitors of serotonin and noradrenaline reuptake have analgesic effects, improve sleep patterns and relax muscles. More recently, anticonvulsants such as gabapentin have been used in the control of SDM ${ }^{(15)}$. In addition to drugs, other invasive and non-invasive therapies are also being used in the treatment of MPS ${ }^{(16)}$. The non-invasive treatment involves the options of ischemic compression (IC), cold spray with stretching, massage, heat thermotherapy, therapeutic ultrasound, low-power laser therapy, therapeutic electrostimulation by transcutaneous electrical neurostimulation (TENS and/or Interferential), passive stretching, among others ${ }^{(17)}$. In the invasive treatments, dry needling (DN) can be used, acupuncture and injection of drug solutions, such as lidocaine, xylocaine and botulinum toxin can be used ${ }^{(18)}$. In order to obtain better treatment results of MPS, many physiotherapists are using IC and DN associated with electrothermotherapeutic and kinesiotherapeutic resources of physiotherapy, without, however, having a consensus on which of these therapies presents the best results ${ }^{(4)}$.

IC is a treatment technique for the active TP, present in the MPS, which consists of manual compression of the active TP in order to obtain its inhibition. It has been argued that this technique restores the length of the sarcomeres and produces an increase in vascularization, with a consequent increase in oxygenation and local hyperemia, producing a gradual relaxation of tension in the TP region without generating additional tension in the other portions of the muscle ${ }^{(19)}$. IC is aimed at decreasing the palpable nodule, muscle fiber tension, pain and tenderness in the $\mathrm{TP}^{(12)}$. IC is a technique of manual therapy, widely used in the clinical practice of physical therapy, which consists in the application of a compressive, gradual mechanical force to active TP for the treatment of MPS ${ }^{(19)}$. In the performance of IC, the physiotherapist finds a tissue barrier that offers resistance, however, with the application of several IC sessions, there may be reduction and even elimination of this barrier, not to mention that the patient's pain threshold increases in each session, facilitating the reception of this therapy. The goal of IC is to decrease palpable nodule, muscle fiber tension, pain and sensibility in the TP. However, the desired effect with the application of IC can be hampered by the intense pain associated with irritation (inflammation) in the active TP, so that patients do not tolerate the application of this technique ${ }^{(10)}$. When the patient's pain threshold is not taken into account or when there is exaggerated compression, we can lead to a condition of increased sensitivity and pain, due to increased tension of the fibers $^{(8)}$.

The DN technique emerged empirically, in cadaver manipulation procedures or under surgical conditions, following the model of manipulation with needles, without associated medication in situations of radiculopathy ${ }^{(20)}$. In the case of needling of active TP in MPS, the needling may be deep or superficial. The DN in TP of specific muscles produce the reduction or elimination of both the referred symptoms and the local sensitivity of the TP, being responsible for the complete elimination of pain in $60.0 \%$ of the patients ${ }^{(15,21)}$. $\mathrm{DN}$ is a relatively new method among the others used to combat pain ${ }^{(22)}$. The needles used in DN treatment are sterile and made of stainless steel, having a copper or aluminum cable and must be flexible to prevent breakage, which may occur due to muscle spasm after insertion. However, prior to insertion of the sterile needle, the region of the body to which it is to be applied should have been cleaned with alcohol or an antiseptic material ${ }^{(23)}$. Prior examination of the needles prior to their insertions is necessary to disable the useless ones, with possible defects ${ }^{(20)}$. The time of application of the DN technique may vary according to the patient's level of pain intensity ${ }^{(24)}$. The efficacy of DN depends on the accuracy of the palpatory location of the TPs correlated with myofascial pain, the proper application of the needling, the number of 
sessions performed and the modification of psychoemotional factors and posture habits by people affected, who must be oriented by the therapist ${ }^{(10)}$. In the absence of this, the treatment may be considered placebo and even cause a more intense pain scenario than that one originally reported by the patients ${ }^{(22)}$. In light of the above, the objective of this study was to compare two types of therapeutic interventions associated to physiotherapy procedures in the treatment of MPS patients.

\section{METHOD}

This study was carried out as a cross-sectional study with a mixed approach, using interventions in subjects in a physiotherapy clinic located in the city of Rio Verde (GO), Brazil. This methodology was chosen to satisfactorily achieve the primary objective of this research. This study was approved without restrictions by the Research Ethics Committee of the University of Rio Verde, Rio Verde, GO, under the CAAE protocol: 53610916.0.0000.5077. The sample consisted of 40 participants. All participants were approached in a standardized way by a trained researcher, received knowledge about the study, to which they were invited to participate, voluntarily consenting to the invitation to sign the free and informed consent form. Participation in the research implied minimal risk to the participants, that is, there was no interference of researchers in any aspect of physical, psychological and social well-being as well as intimacy, according to the parameters contained in Resolution n. 466, dated December $12^{\text {th }}, 2012$, from the Brazilian National Health Council, which rules research involving human beings. The informed consent document was signed for publication of all patient participants. In the selection of participants, inclusion criteria were: age - 20 to 60 years old, people of both genders, with clinical diagnosis of MPS and complaint of local or referred pain in several body portions, for a time superior to six weeks, with the presence of at least four active TP, identified through palpable tense band, referred pain, twitch response and jump sign through trigger point compression $^{(8,25)}$. The exclusion criteria were: pregnant women, patients with clinical evidence of organic disorders such as renal disease, coronary artery syndrome, osteoporosis, arthralgia, bursitis, tendinopathies, ligamentopathies, discopathies, people who were under musculoskeletal medication or performing complementary therapy to those in this study. In addition, those who did not sign the free and informed consent form were also excluded. The data collected were: identification, sex, age, weight, height, approximate time of manifestation of pain, active TP and location of pain. The 40 patients were divided into two groups, consisting of 20 participants in each. The groups were matched for variables: age, body mass index, number and location of TPs, sex, time of pain manifestation and posture. The first group, ischemic compression and physiotherapy (ICF) received IC and physiotherapy (therapeutic ultrasound - UST, TENS, massotherapy and stretching) in active TP. The IC technique consisted of applying a compression, made by the therapist's thumb, with incremental force increase in the active TP, for 90 seconds, in such a way that the initial compression sensation was transformed into pain and, after its relief, the compression was increased until the pain was again reported ${ }^{(15,26)}$. TENS, with a frequency of $10 \mathrm{~Hz}$ and a pulse width of $230 \mu \mathrm{s}$, was used for 25 minutes and the UST calibrated with $1 \mathrm{MHz}$ wave emission in continuous mode, with a dosage of $1.0 \mathrm{~W} / \mathrm{cm}^{2}$ for 5 minutes in the active $\mathrm{TP}^{(14)}$. At the end of each treatment, massotherapy and passive stretching were performed for 20 seconds in the muscles that presented the active TP. The second group, dry needling and physiotherapy (DNF) received DN and physiotherapy (UST, TENS, massotherapy and stretching) in the active $\operatorname{TP}^{(15,27)}$. Initially, the most sensitive portion of the active TP was palpated and then a needle of $0.26 \mathrm{~mm} \times 25 \mathrm{~mm}$ was inserted perpendicular to the skin and towards the tight band, composed of the contracted muscle fibers, until the moment a first contractile response appeared on the spot. The needles were inserted and withdrawn until there were three involuntary muscle contractions, making an average time of two minutes at each point. The application of TENS, UST, massotherapy and passive stretching followed the same parameters mentioned in the treatment of the ICF group. The periodization and time of the treatment sessions were performed three times a week, on alternate days, for 50 minutes, for a total of 10 sessions $^{(1)}$. Prior to the application of the therapy the skin was cleaned with $70.0 \%$ alcohol in the non-dominant forearm, and the individuals were positioned with the forearm in an anatomical position and the elbow in 90 o flexion. The TENS was applied for 20 minutes at the $100 \mathrm{~Hz}$ frequency, pulse duration of $40 \mu \mathrm{s}$ and intensity at the sensory limit using the Endomed 582 ID apparatus (Enraff Nonius, Holland) with rectangular silicone / carbon electrodes measuring $8 \mathrm{~cm} \times 6 \mathrm{~cm}$, longitudinally positioned over the anterior region of the forearm ${ }^{(1)}$. Two researchers, previously trained, were involved to perform, isolatedly, orientation, evaluation and treatment of the participants of both groups. It is worth considering that the participants' evaluation took place in two moments: before the start of the treatment and one week after the last session, without the evaluator being aware of the treatment that was destined to the participants of both groups.

This study used statistical analysis, including proportions for categorical variables, significance and standard deviation for continuous variables. In order to evaluate the change in perception of pain and quality of life of the participants, the Student's $t$ test $(p<0.05)$ was applied for paired samples, for the intragroup comparison and for the independent samples between the groups, evaluating the before and after of the therapeutic.

For so, the instrument Visual Analog Scale (VAS) was used, which presents the objective of assisting in the 
process of verification of pain intensity. This scale indicates the efficiency of a determined treatment based on the improvement or worsening of the pain ${ }^{(28)}$. The questionnaire for QOL assessment applied in this study was developed by the Mental Health Program of the World Health Organization in Geneve through the WHOQOL (Quality of Life Group) in 1998 and validated for Brazil Researchers by Marcelo Pio de Almeida et al., in $2000^{(29)}$. The authors of this research use the WHOQOL-bref (26 questions), an abbreviated version, in order to turn the application easier, shortening the filling time and, thus, achieving the proposed goal.

These two evaluation methods were chosen for referring to scientifically validated instruments that were easy to be applied and understood by the patients, as well as very successful in research involving a variety of treatments in MPS; considering, respectively, the evaluation of the pain and quality of life of the participants. It is worth highlighting that in data collection and analysis there was no kind of intercurrence that could influence the study results.

\section{RESULTS}

The clinical and demographic data related to the 40 patients in this study, subdivided into the ICF group and the DNF group of 20 participants each, are described in Table 1.

The most affected muscles in the participants of the ICF and DNF groups by the presence of active trigger points, in percentage values, are thus attributed: $1.9 \%$ for vastus medialis, soleus, rectus femoris, pectoralis major, infraespinatus inferior, gastrocnemius, femoral biceps adductor; 3.7\% for supraspinatus, squalane and sternocleidomastoid; $5.6 \%$ for rhomboid and iliopsoas; 7.4\% for trapezius, suboccipital, large dorsal and scapula lift; 9.3\% for infants; $11.1 \%$ for the gluteus medius; And 13.0\% for lumbar spine. The Student's $t$ test was used to analyze the VAS results of the participants in both groups, before starting treatment and one week after finishing it. There was a statistically significant reduction in the VAS scores of participants treated in both groups, ICF group (10.005 Student's $t$-test) and DNF group (18.548 Student's $t$-test), with a greater reduction in VAS scores in the DNF group, which demonstrates the effectiveness of the application of physical therapy associated with IC and physiotherapy applied to DN in the treatment of patients with MPS, being physiotherapy associated to DN promoted better results in pain control (Table 2).

Regarding the WHOQOL-bref questionnaire, there was a significant improvement only in the social domains before and after the ASF (-2.727), in the environmental domain before and after the ICF (-2.519) and DNF (-2.330), and in quality of life, before and after ICF (-2.798) and DNF (-3.096), analyzed before and one week after treatment. This shows that there was an improvement in the social performance of the participants treated with DNF, environmental of the participants treated with ICF and DNF, and the quality of life of participants treated with ICF and DNF, as shown in Table 3.

In this study, an association of classical physiotherapy with IC and DN was used in the treatment of patients with MPS, with positive evidence in both treatments, in pain relief and in the improvement of patients' quality of life. The group treated with physiotherapy and DN presented the best results. In a randomized controlled trial comparing injection methods in myofascial pain syndrome, no differences in pain intensity were observed between the application of $2 \mathrm{ml}$ of lidocaine at $1.0 \%$ and DN in active TP, in which patients were assessed after 4 and 12 weeks $^{(30)}$. In another study, no differences were observed in the assessment of pain intensity in patients with active TP in cervical muscles, with headache who underwent $0.5 \%$ lidocaine, DN and botulinum toxin, evaluated after 1.4 and 12 weeks $^{(18)}$. Yet, in a study on myofascial TP injection for groin pain in patients with chronic prostatitis / chronic pelvic pain syndrome, pain relief was observed in patients after application of $1.0 \%$ lidocaine followed by IC in active $\operatorname{TP}^{(20)}$.

$I C$ and DN are techniques that are increasingly being used by physiotherapists in the treatment of MPS, due to their analgesic, circulatory, muscle relaxation and functional benefits, not to mention that these techniques are easy to apply, inexpensive and present more effective and faster results than classical physiotherapy ${ }^{(1)}$. The use of only classical physical therapy resources in the treatment of MPS may be unsatisfactory in the control of the pain of people affected, and the effects of these procedures usually occur through

Table 1. Clinical and demographic data of participants of ICF e DNF groups

\begin{tabular}{lccccccc}
\hline \multirow{2}{*}{ Pair } & \multirow{2}{*}{ Variable } & \multicolumn{3}{c}{ Paired Differences } & \multirow{2}{*}{ S } & gl & Sig. (bilateral) \\
\cline { 3 - 6 } & & Average & Standard Deviation & Standard average mistake & \\
\hline Pair 1 & Age & -3.00 & 19.26 & 4.30789 & -0.696 & 19 & 0.495 \\
Pair 2 & Weight & -9.30 & 27.62 & 6.17725 & -1.506 & 19 & 0.149 \\
Pair 3 & Height & -0.01 & 0.148 & 0.03313 & -0.377 & 19 & 0.710 \\
Pair 4 & Body mass index & -2.08 & 8.64 & 1.93354 & -1.079 & 19 & 0.294 \\
Pair 5 & Pain manifestation & -7.30 & 45.99 & 10.28440 & -0.710 & 19 & 0.486 \\
Pair 6 & Trigger points $(\mathrm{n})$ & 0.50 & 5.18 & 1.15963 & 0.431 & 19 & 0.671 \\
\hline
\end{tabular}

Note: Pain manifestation in time; Sig=significance. 
Table 2. Comparison between the visual analogue scale before and one week after the treatment in the groups of dry needling and physiotherapy and of ischemic compression and physiotherapy of the participants of the ICF and DNF

\begin{tabular}{|c|c|c|c|c|c|}
\hline \multirow{2}{*}{ Comparative } & \multicolumn{2}{|c|}{ Paired Differences } & \multirow{2}{*}{$t$} & \multirow{2}{*}{ gl } & \multirow{2}{*}{ Sig. (bilateral) } \\
\hline & Average & Standard Deviation & & & \\
\hline ICF VAS before and ICFF VAS after & 6.05 & 2.70 & 10.005 & 19 & $0.000^{* *}$ \\
\hline DNF VAS before and DNF VAS after & 7.80 & 1.88 & 18.548 & 19 & $0.000 * *$ \\
\hline
\end{tabular}

Note: ICF VAS before=VAS before sessions in the ischemic compression and physiotherapy group; ICF VAS after=VAS one week after the sessions in the ischemic compression and physiotherapy group; DNF VAS before=VAS before sessions in the dry needling and physiotherapy group; DNF VAS after=VAS one week after the sessions in the group dry needling and physiotherapy. ${ }^{* *}$ Significant at $5.0 \%$ probability by Student's t-test.

Table 3. Comparison of WHOQOL-Bref domains applied before and one week after treatment in the two groups of dry needling and physiotherapy and of ischemic compression and physiotherapy of the participants of the ICF and DNF groups

\begin{tabular}{|c|c|c|c|c|c|}
\hline \multirow{2}{*}{ Comparative } & \multicolumn{2}{|c|}{ Paired differences } & \multirow{2}{*}{$t$} & \multirow{2}{*}{ gl } & \multirow{2}{*}{ Sig. (bilateral) } \\
\hline & Average & Standard Deviation & & & \\
\hline PD ICF before and PD ICF after & 3.57 & 25.35 & 0.63 & 19 & 0.536 \\
\hline PD DNF before and PD DNF after & 1.67 & 24.45 & 0.306 & 19 & 0.763 \\
\hline PSD ICF before and PSD ICF after & -2.91 & 30.75 & -0.424 & 19 & 0.676 \\
\hline PSD DNF before and PSD DNF after & -4.06 & 17.16 & -1.059 & 19 & 0.303 \\
\hline SD ICF before and SD ICF after & -0.45 & 21.37 & -0.094 & 19 & 0.926 \\
\hline SD DNF before and SD DNF after & -11.78 & 19.32 & -2.727 & 19 & $0.013^{* *}$ \\
\hline ED ICF before and ED ICF after & -8.53 & 15.15 & -2.519 & 19 & $0.021 * *$ \\
\hline ED DNF before and ED DNF after & -10.83 & 20.78 & -2.33 & 19 & $0.031^{* *}$ \\
\hline QL ICF before and QL ICF after & -8.13 & 12.99 & -2.798 & 19 & $0.011^{* *}$ \\
\hline QL DNF before and QL DNF after & -9.82 & 14.19 & -3.096 & 19 & $0.006 * *$ \\
\hline
\end{tabular}

Note: PDICF before and after: physical domain before and one week after the sessions in the ischemic compression and physiotherapy group; PD DNF before and after: physical domain before and one week after the sessions in the group dry needling and physiotherapy; PHD ICF before and after: psychological domain before and one week after the sessions in the ischemic compression group; PHD DNF before and after: psychological domain before and one week after the sessions in the group dry needling and physiotherapy; SD ICF before and after: social domain before and one week after the sessions in the ischemic compression group; SD DNF before and after: social domain before and one week after the sessions in the group dry needling and physiotherapy; ICF before and after: environmental domain before and one week after the sessions in the ischemic compression group; DNF before and after: environmental domain before and one week after the sessions in the group dry needling and physiotherapy; QL ICF before and after: quality of life before and one week after the sessions in the ischemic compression group; QL DNF before and after: quality of life before and one week after the sessions in the group dry needling and physiotherapy.

** Significant at $5.0 \%$ probability by Student's t-test.

the attendance of many sessions, demanding time and high cost $^{(14)}$. When comparing the effects of DN and IC associated with passive stretching in patients with MPS, pain relief and improvement of patients' QOL in both treatment situations can be verified, with patients treated with DN and passive stretching show the best results ${ }^{(1)}$. Significant pain relief in patients with MPS treated with IC and passive stretching compared to the control group, shows that this therapeutic may require a larger number of sessions, around 5 or 6 to cause the deactivation of the TGs and generate analgesia ${ }^{(19,31)}$. DN presents good results in the treatment of MPS, favoring improvement of patients' pain and $\mathrm{QOL}^{(15,17,24,32)}$. These authors comment on the possibility of DN being applied together with other therapies such as drugs, physical therapy, manual therapy techniques with an emphasis on IC, physical activity and postural corrective techniques to maximize the treatment results and minimize the discomfort generated by DN.

It was thought that the combination of TENS, UST, massotherapy and passive stretching, in both groups, at the end of the DN and IC, which constitute the analgesic resources, of circulatory effect and muscle relaxation, to minimize the unpleasant effects of these techniques and increase therapeutic benefits. The reassessment of DN-treated participants after the minimum period of one week was also suggested, for this procedure usually causes local pain, edema and local irritation, due to the effect of the needling, and, in the short term, provides a false impression of aggravation of the pain.

\section{CONCLUSION}

Taking into account the experimental conditions of this study, it can be concluded that physiotherapy associated with IC as well as physiotherapy associated with DN can promote pain relief and improve the QOL of patients with MPS. Participants undergoing physiotherapy associated with DN presented a more accentuated reduction in pain intensity, showing that DN promoted better analgesic benefits than IC in MPS patients. 


\section{AUTHORS CONTRIBUTIONS}

VRMP: elaborated the study design, performed the critical intellectual revision of the manuscript. GMP: elaborated the study design, performed the data collection. JJ: performed the statistical analysis. CFS: performed the data collection. HMS: performed the statistical analysis, performed the critical intellectual revision of the manuscript. All authors read and approved the final manuscript.

\section{CONFLICT OF INTEREST}

The authors declare that there is no conflict of interest.

\section{AUTHORS DETAILS}

'Universidade de Rio Verde - UniRV - Rio Verde (GO), Brazil.

${ }^{2}$ Universidade Federal do Amazonas - UFAM - Parintins (AM), Brazil.

\section{REFERENCES}

1. Santos RBC, Carneiro MIS, Oliveira DM, Maciel ABR, Silva KKM, Araújo MGR. Impact of dry needling and ischemic pressure in the myofascial syndrome: Controlled clinical trial. Fisio Mov. 2014; 4: 515-22.

2. Musse, A. Síndrome dolorosa miofascial. In: Lianza S, editor. Medicina de reabilitação. 3rd ed. Rio de Janeiro: Guanabara Koogan; 1995. p. 163-77.

3. Yeng LT, Kaziyama HH, Teixeira MJ. Síndrome dolorosa miofascial e fibromialgia. In: Siqueira JT, Teixeira MJ, editores. Dor orofacial diagnóstico, terapêutica e qualidade de vida. São Paulo: Maio; 2001. p. 405-30.

4. Asher SN. Pontos-gatilhos: uma abordagem concisa. São Paulo: Manole; 2008. 208 p.

5. Weschenfelder V, Agne JE. Efeitos da hidrocinesioterapia no tratamento da Síndrome Dolorosa Miofascial: um estudo de caso. Rev. Digital. 2007; 106: $1-2$.

6. Davies C, Davies, A. Livro terapêutico do ponto-gatilho: guia de tratamento da dor. 2nd ed. São Paulo: Roca; 2012. 448 p.

7. Aranha MF, Alves MC, Bérzin F, Gavião MB. Efficacy of electroacupuncture for myofascial pain in the upper trapezius muscle: a case series. Rev Bras Fisioter. 2011; 15(5): 371-9.

8. Simons DG, Travell T, Simons LS. Myofascial pain and dysfunction: The trigger point manual. 2nd ed. Vol. 1, Upper half of body. Baltimore: Williams \& Wilkins; 1999. 1038 p.

9. Wolens D. The myofascial pain syndrome: A critical appraisal. Am Phys Med Rehabil. 1998; 12(2): 299-316.

10. Abbaszadeh-Amirdehi M, Ansari NN, Naghdi S, Olyaei G, Nourbakhsh MR. The neurophysiological effects of dry needling in patients with upper trapezius myofascial trigger points: Study protocol of a controlled clinical trial. BMJ Open. 2013; 3(5):e002825.

11. Dommerholt J, Huijbregts P. Myofascial trigger points: Pathophysiology and evidence-informed diagnosis and management. Canadian: Jones \& Bartlett Learning; 2011. 296 p.

12. Dixon MW. Massagem miofascial. Rio de Janeiro: Guanabara Koogan; 2007.

13. Leitão A, Leitão VA. Síndrome miofascial. In: Musse CA, editor. Clínica de reabilitação. São Paulo: Atheneu; 1995. p. 116-125.

14. Rayegani SM, Bayat M, Bahrami MH, Raeissadat SA, Kargozar E. Comparison of dry needling and physiotherapy in treatment of myofascial pain syndrome. Clin Rheumatol. 2014; 33(6): 859-64.
15. Campa-Moran I, Rey-Gudin E, Fernández-Carnero J, Paris-Alemany A, Gil-Martinez A, Lerma Lara S, et al. Comparison of dry needling versus orthopedic manual therapy in patients with myofascial chronic neck pain: A single-blind, randomized pilot study. Pain Res Treat. 2015; 2015:327307.

16. Akamatsu FE, Ayres BR, Saleh SO, Hojaij F, Andrade M1, Hsing WT, et al. Trigger points: An anatomical substratum. Biomed Res Int. 2015; 2015:623287.

17. Llamas-Ramos R, Pecos-Martín D, Gallego-Izquierdo T, Llamas-Ramos I, Plaza-Manzano G, Ortega-Santiago R, et al. Comparison of the shortterm outcomes between trigger point dry needling and trigger point manual therapy for the management of chronic mechanical neck pain: A randomized clinical trial. J Orthop Sports Phys Ther. 2014; 44(11): 852-61.

18. Venancio RA, Alencar FG Jr, Zamperini C. Botulinum toxin, lidocaine, and dry-needling injections in patients with myofascial pain and headaches. Cranio. 2009; 27(1): 46-53.

19. Hou CR, Tsai LC, Cheng KF, Chung KC, Hong CZ. Immediate effects of various physical therapeutic modalities on cervical myofascial pain and trigger-point sensitivity. Arch Phys Med Rehabil. 2002; 83(10): 1406-14.

20. Kim DS, Jeong TY, Kim YK, Chang WH, Yoon JG, Lee SC. Usefulness of a myofascial trigger point injection for groin pain in patients with chronic prostatitis/chronic pelvic pain syndrome: A pilot study. Arch Phys Med Rehabil. 2013; 94(5): 930-6.

21. Junqueira MS. Influência da dor miofascial na enxaqueca e na cefaléia do tipo tensional. Mig Cef. 2006; 9(2): 41-4.

22. Vulfsons S1, Ratmansky M, Kalichman L. Trigger point needling: Techniques and outcome. Curr Pain Headache Rep. 2012;16(5): 407-12.

23. Tough EA, White AR. Effectiveness of acupuncture/dry needling for myofascial trigger point pain. Phys Ther Rev. 2011; 16(2): 147-54.

24. Cerezo-Téllez E, Torres-Lacomba M, Mayoral-Del Moral O, SánchezSánchez B, Dommerholt J, Gutiérrez-Ortega C. Prevalence of Myofascial Pain Syndrome in chronic non-specific neck pain: A population-based cross-sectional descriptive study. Pain Med. 2016; 17(12): 2369-2377.

25. Hong $\mathrm{CZ}$. Lidocaine injection versus dry needling to myofascial trigger point. The importance of the local twitch response. Am J Phys Med Rehabil. 1994;73(4): 256-63.

26. Montañez-Aguilera FJ, Valtueña-Gimeno N, Pecos-Martín D, ArnauMasanet R, Barrios-Pitarque C, Bosch-Morell F. Changes in a patient with neck pain after application of ischemic compression as a trigger point therapy. J Back Musculoskelet Rehabil. 2010; 23(2): 101-4.

27. Hong CZ, Kuan TS, Chen JT, Chen SM Referred pain elicited by palpation and by needling of myofascial trigger points: A comparison. Arch Phys Med Rehabil. 1997; 78(9): 957-60.

28. Bijur PE, Silver W, Gallagher EJ. Reliability of the visual analog scale for measurement of acute pain. Acad Emerg Med. 2001; 8(12): 1153-7.

29. Fleck MP, Louzada S, Xavier M, Chachamovich E, Vieira G, Santos L, Pinzon V. [Application of the Portuguese version of the abbreviated instrument of quality life WHOQOL-bref]. Rev Saude Publica. 2000; 34(2): 178-83.

30. Ay S, Evcik D, Tur BS. Comparison of injection methods in myofascial pain syndrome: a randomized controlled trial. Clin Rheumatol. 2010; 29(1): 19-23.

31. Hanten WP, Olson SL, Butts NL, Nowicki AL. Effectiveness of a home program of ischemic pressure followed by sustained stretch for treatment of myofascial trigger points. Phys Ther. 2000; 80(10): 997-1003.

32. Cagnie B, Castelein B, Pollie F, Steelant L, Verhoeyen H, Cools A. Evidence for the use of ischemic compression and dry needling in the management of trigger points of the upper trapezius in patients with neck pain: A systematic review. Am J Phys Med Rehabil. 2015; 94(7): 573-83. 